\title{
Les figures au collège : un objet didactique complexe
}

The Rhetorical Figures in Secondary School: A Complex Learning Object

\section{Nicole Biagioli}

\section{(2) OpenEdition}

\section{Journals}

\section{Édition électronique}

URL : http://journals.openedition.org/pratiques/2570

DOI : $10.4000 /$ pratiques. 2570

ISSN : 2425-2042

Éditeur

Centre de recherche sur les médiations (CREM)

\section{Référence électronique}

Nicole Biagioli, «Les figures au collège : un objet didactique complexe », Pratiques [En ligne], 165-166। 2015, mis en ligne le 01 octobre 2015, consulté le 14 novembre 2019. URL : http://

journals.openedition.org/pratiques/2570; DOI : 10.4000/pratiques.2570

Ce document a été généré automatiquement le 14 novembre 2019.

(c) Tous droits réservés 


\title{
Les figures au collège: un objet didactique complexe
}

The Rhetorical Figures in Secondary School: A Complex Learning Object

\author{
Nicole Biagioli
}

\section{Introduction}

1 Les figures au collège : le choix du niveau d'études est provocateur et historiquement significatif. La classe de rhétorique qui correspondait à l'actuelle classe de $1^{\text {re }}$ des lycées généraux était le fleuron de l'enseignement secondaire quand celui-ci était réservé à une élite. On devine du coup que la complexité visée n'est pas seulement de l'ordre de la dénotation (qu'appelle-t-on figures? quelles structures linguistiques - chaine parlée, syntaxe, lexique, énoncé - concernent-elles ? appartiennent-elles à la langue et/ou au discours ?), mais aussi de la connotation. Réduites dans le programme à une douzaine d'items $^{1}$, les figures continuent à occuper autant sinon plus de place dans l'imaginaire scolaire, mais leur rôle emblématique s'est désormais clivé.

D'un côté, elles représentent l'aboutissement des apprentissages littéraires. Rapportant le récit par Gracq de sa première demi-heure en classe de rhétorique, durant laquelle le professeur avait donné à analyser la phrase de La Bruyère « À mesure que la faveur et les grands biens se retirent d'un homme, ils laissent voir en lui le ridicule qu'ils couvraient et qui y était sans que personne s'en aperçût » sans qu'aucun élève ne repère la métaphore de la marée, A. Boissinot (2001: 10), ne retient que le souvenir positif laissé au futur écrivain par la " petite révélation » de la solution. Il ne cache pas son admiration pour " un enseignement qui conjuguait heureusement art de lire et art d'écrire», pour lequel le texte n'est pas seulement "objet d'admiration passive», mais "déclenche une démarche d'appropriation, de voracité ». D'un autre côté, les figures paraissent un objet didactique daté, quasiment obsolète, chichement dosé pour éviter d'ennuyer les élèves : «Sans introduire de manière systématique une connaissance prématurée de la rhétorique, on peut en exploiter quelques éléments, notamment dans le domaine des 
figures, en les inscrivant toujours dans la perspective globale du discours et de sa visée " lit-on dans l'Accompagnement du programme de $3^{\mathrm{e}}$ (1999: 171). Cette position médiane, entre lecture et écriture, langue et langage, littérature et discours quotidien, serait-elle le secret de leur étonnante résistance?

Pour répondre à cette question, nous organisons notre étude en deux parties. La

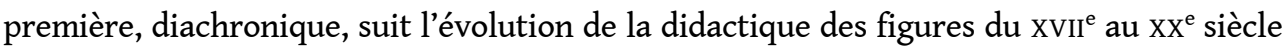
en parallèle avec la mise en place de l'institution scolaire.

4 La seconde partie, synchronique, montre quelle place les figures occupent dans l'enseignement actuel du français au collège en France, en termes de contenus, de progressions didactiques et de stratégies d'enseignement.

\section{Historique}

5 Les figures font partie à la fois de l'histoire de l'école et de celle de la formation professionnelle. Si l'éloquence, née de l'éclosion dans la Grèce du v viècle des notions de justice et de démocratie, a permis de prendre conscience du fonctionnement des figures et de les classer, elle a aussi ouvert leur application à d'autres champs professionnels que la justice ou la politique, à commencer par celui de leur enseignement. Certains habitus professionnels des rhéteurs antiques ont perduré jusqu'à l'époque moderne. Ce sont :

- la conscience de la transversalité des compétences rhétoriques. Jusqu'à la seconde moitié du $\mathrm{XVII}^{\mathrm{e}}$ siècle l'expression "belles-lettres» recouvre aussi bien les sciences, le droit, la médecine que les lettres (Viala, $1985: 28)$;

- la tendance à associer les figures à la seule élocution bien qu'elles interviennent aussi dans l'invention, en stimulant l'imaginaire des auteurs (Mauron, 1963), et dans la composition ;

- le choix du discours comme champ d'apprentissage au détriment de la langue, avec deux conséquences :

- l'usure des figures se voit légitimée dans certains usages, car la nouveauté d'une figure peut faire obstacle à la communication,

- les tropes ne sont abordés que lorsque le discours les remotive. Il faut attendre P. Fontanier (au XVIII ${ }^{\mathrm{e}}$ siècle) pour que la rhétorique fasse une place (secondaire) à la catachrèse (Genette, $1977: 11)$.

\subsection{Les figures dans les traités de rhétorique}

6 Il est difficile d'expliquer la place occupée par les figures dans l'histoire de la discipline «français » si l'on oublie que leur enseignement est à la fois un effet et un facteur de l'institutionnalisation de la littérature à l'époque classique :

«Si le cursus scolaire restait accaparé par le latin et les humanités, la littérature française gagnait du terrain à la périphérie. Son progrès est lié aux débuts d'un enseignement en langue française qui s'amorça alors [...] et pour lequel les maîtres de Port-Royal jouèrent un rôle d'avant-garde. Elle se manifesta aussi dans l'étude de la rhétorique, un des piliers du programme de formation : La Rhétorique française de l'oratorien Bernard Lamy marque à cet égard une étape importante, les oratoriens étant après les jésuites l'ordre le plus actif par ses collèges. Et, dans les collèges jésuites, les exercices complémentaires de l'enseignement de base [...] firent de plus en plus de place au français dans la seconde moitié du siècle » (Viala, 139-140).

7 La littérature a fait son entrée à l'école par les exemples des manuels de rhétorique. Les figures apparaissent d'emblée comme un facteur de légitimation de la profession 
littéraire. De par ses fonctions d'enseignant de grammaire, rhétorique et philosophie et sa triple qualité de mathématicien, philosophe et physicien, B. Lamy (1640-1715) était particulièrement bien placé pour établir les bases d'une didactique moderne de la rhétorique. Dans la préface de La Rhétorique ou l'art de parler (1675), il valorise l'imprégnation mimétique et le réemploi : "Une rhétorique peut être bien faite, sans qu'on en retire du fruit, lorsqu'à la lecture de ses préceptes on ne joint point celle des orateurs, et l'exercice (XIII)». Ceci le conduit à choisir un mode de citation extensif qui respecte l'intégrité des textes et facilite leur compréhension :

«Il faut donc que les Maîtres fassent lire à leurs Disciples les excellentes pièces d'éloquence, \& qu'ils ne se servent de la Rhétorique que pour leur faire remarquer les traits éloquents des auteurs qu'ils leur font voir; ce qui ne peut bien se faire qu'en lisant les pièces tout entières. Les parties détachées, qu'on en propose pour exemple, perdent les graces quand elles sont hors de leur place : séparées du reste du corps elles sont, pour ainsi dire, sans vie (XXII)».

8 Les figures constituent un point névralgique de la didactique du discours. On ne peut les repérer sans modélisation préalable, ce qui exige des exemples brefs, limités à l'illustration des définitions. En même temps, on ne peut les pratiquer sans les réinvestir en situation, dans la lecture-écriture d'énoncés authentiques. L'oscillation, pour les supports d'enseignement, entre la forme du dictionnaire et celle du traité, et pour les objectifs, entre maitrise de la langue et maitrise du discours, est déjà perceptible. Toutefois Lamy ne s'inscrit pas dans une aire linguistique particulière. Au plan pratique, il nourrit les compétences plurilingues de ses élèves en entremêlant les originaux latins et grecs avec leurs traductions françaises et avec des originaux français. Au plan théorique, situant les figures au seul niveau du discours, il ne doute pas de la nature transdiscursive et translinguistique de son propos : «Mon Ouvrage, comme je l'ai insinué, ne regarde pas seulement les Orateurs, mais généralement tous ceux qui parlent et qui écrivent, les Poètes, les Historiens, les Philosophes, les Théologiens. Quoique j'écrive en français, j'espère que mon travail sera utile dans toutes les langues » (ibid.).

9 La posture énonciative est proche de celle de nos manuels. B. Lamy s'adresse à l'élève tout en visant les pratiques du maitre : « Cet Ouvrage sera donc utile aux jeunes gens qu'il faut accoutumer à aimer la vérité, \& à consulter la raison pour penser et agir selon sa lumière » (ibid.). Il ne sort pas du cadre institutionnel : catholique (nombreuses références aux Écritures et aux Pères de l'Église), préprofessionnel (allusions aux professions libérales ou religieuses auxquelles il forme ses élèves), dogmatique (à preuve les titres des chapitres 4 : «les tropes doivent être clairs» et 8 «Les figures sont utiles et nécessaires »), axiomatisé (traitant des causes: l'appareil phonatoire et les langues naturelles au chapitre 1, avant l'effet : les tropes, au chapitre 2). Il faut donc, si l'on veut observer les premières traces de réflexion sur la démocratisation de la rhétorique, se tourner vers des traités qui s'adressent à d'autres publics que le public scolarisé.

10 Gabriel Henri Gaillard (1726-1806) avait 19 ans lorsqu'il a publié sa Rhétorique française à l'usage des jeunes demoiselles en 1745 . Son livre renvoie tout en les résumant aux ouvrages de ses prédécesseurs. Forme et contenu sont pensés en fonction du public. Le vocabulaire est simplifié et le catalogue exhaustif des figures remplacé par une sélection lestement assumée :

«Il y a plusieurs autres figures; tant de pensées que de mots; mais leur peu d'usage, leur inutilité, leurs noms rudes et barbares nous ont déterminé à les laisser dans l'oubli, où il est à propos qu'elles demeurent ensevelies » (403). 
11 Comparée à celle de B. Lamy : « La périphrase est un détour que l'on prend pour éviter de certains mots qui ont des idées choquantes, \& pour ne pas dire de certaines choses qui produiraient de mauvais effets » (169), la définition de la périphrase chez Gaillard : «La périphrase est une figure fort ordinaire, surtout aux poètes, qui s'en servent pour étendre et enrichir une idée qui eût pu être exprimée plus simplement, mais avec beaucoup moins de noblesse » (393) est plus pédagogique et démystifie la figure tout en précisant sa sphère d'emploi.

Dans un système d'éducation différencié, ce type de transposition didactique s'applique aux acteurs sociaux que l'on destine à des tâches intermédiaires entre l'exécution et la décision, i.e. à être des auxiliaires compétents, ce qui est le cas des femmes au XVIII ${ }^{\mathrm{e}}$ siècle. J.-J. Rousseau en formule le principe dans Émile (1762) :

«La recherche des vérités abstraites et spéculatives, des principes, des axiomes dans les sciences, tout ce qui tend à généraliser les idées, n'est point du ressort des femmes : leurs études doivent se rapporter toutes à la pratique; c'est à elles de faire l'application des principes que l'homme a trouvés, et c'est à elles de faire les observations qui mènent l'homme à l'établissement des principes » (736).

13 Cet argumentaire réapparaitra à chaque étape de la scolarisation des filles, mais aussi du développement de l'école obligatoire, primaire, puis secondaire.

Les textes d'appui sont choisis en fonction du lectorat. Ils sont contemporains (tragédies de Voltaire, poèmes de J.-B. Rousseau), mettant en scène des femmes (héroïnes tragiques), ou écrits par des femmes (Gaillard semble vouer un culte à Madame Deshoulières). Les références sont minimales : noms de l'auteur et de l'œuvre quand elle est connue. Que les femmes n'aient pas d'avenir professionnel a donc infléchi leur formation rhétorique vers l'acquisition d'une compétence de lecture experte généraliste, car ${ }_{20}{ }_{0}$ c'est la principale

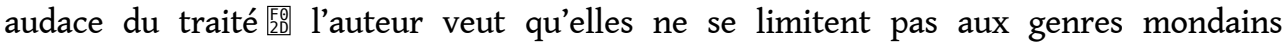
appréciés des salons, mais abordent les genres sérieux: tragédie, épopée, plaidoyer, sermon. À l'instar des manuels modernes, c'est donc le programme de lecture qui décide de la progression en pratique de l'écrit.

Les femmes n'écrivant pas de traité, ni de plaidoirie, mais des lettres, la lecture occupe une place beaucoup plus importante que l'écriture. De plus comme les règles de l'écriture épistolaire relèvent plus de la politesse que de la littérature, Gaillard part des situations d'emploi des figures et non de leur analyse. De même qu'il part du langage ordinaire pour faire apprécier leurs emplois littéraires :

«Dans presque toutes les langues, il y a une infinité de choses et de modifications de chose qui manquent de termes propres; d'autres en ont, mais ils ne sont point assez énergiques. Cette disette de mots a obligé de recourir à des tours étrangers ; de là est venu l'origine des tropes [...], on s'en est servi dans la suite autant et plus pour le simple ornement que pour la nécessité » (377).

On retrouve ce parti pris dans les manuels de collège actuels qui tous font précéder les sections consacrées aux figures d'une leçon sur sens propre/sens figuré, quand ils ne s'en contentent pas.

17 Les figures occupent le chapitre 6 du livre III, consacré à l'élocution, et sont réparties en figures de pensée (qui ne changent pas si on change les mots: exemple: antithèse, apostrophe, description), figures de mots (qui reposent sur le changement de sens: métaphore, allégorie, hyperbole), et figures de mots qui ne sont point tropes (mais sont pris dans leur sens habituel : périphrase, répétition). L'originalité de ce traité réside dans la longueur et la multiplication des exemples (quatre pour la description), que l'auteur 
invite explicitement à comparer (sans toutefois le faire, 299). Il marque un momentum, celui où la rhétorique cesse d'être un objet didactique à part entière et se voit subordonnée à l'étude de la littérature.

\subsection{Figures et enseignement du français}

18 Aux XIX et $\mathrm{XX}^{\mathrm{e}}$ siècles, la rhétorique subit dans les instructions officielles une alternance de dépréciations et de réhabilitations, sans qu'on cesse pour autant d'étudier les figures. Elles apparaissent cependant comme le maillon faible du système. Elles occupent plus de place dans les traités que l'invention, la composition et la prononciation réunies, mais le niveau microstructurel auquel on les cantonne fait qu'on les considère comme secondaires. C'est pourquoi il ne faut pas se limiter aux effets de surface du combat idéologique, et tâcher au contraire d'approfondir les raisons de leur résistance.

19 Se fondant sur l'observation des devoirs d'élèves, V. Houdart-Mérot (26) «invite à nuancer l'idée répandue de la mutation pédagogique de 1880 , de la rhétorique à la critique, de l'élocution à l'invention ». L'étude des traités de rhétorique confirme ce constat et contredit en même temps une autre idée préconçue, celle de l'assimilation de la tradition rhétorique à l'enseignement jésuite. En effet, dans ses Principes de littérature (1882), le Père Mestre, S. J., défend, dès le titre, la nécessité d'enseigner la littérature, et la littérature française moderne.

Regrettant que l'histoire littéraire ne figure qu'au programme de la première et s'oublie une fois passé le baccalauréat, il veut en débuter l'apprentissage en seconde et coupler son étude avec celle de la rhétorique. Mais celle-ci n'occupe que la première partie consacrée aux Principes généraux de l'art d'écrire dont le deuxième chapitre est consacré aux figures, ornements du style, tandis que la deuxième partie intitulée Composition présente les Principaux genres en prose, et qu'une troisième partie intitulée Poésie (entendue au sens large, par opposition à la prose) détaille les genres poétiques principaux (épique, lyrique, dramatique) et secondaires (satire, madrigal).

21 On voit donc une nouvelle catégorie, le genre, succéder aux figures pour assurer la médiation entre littérature et rhétorique. Toutefois les définitions des genres ne sont exemplifiées que par les biographies des auteurs antiques et modernes qui s'y sont illustrés, les échantillons textuels restant cantonnés dans la partie consacrée au style et aux figures. Le plan de l'ouvrage renverse l'ordre traditionnel. L'élocution vient en premier, l'étude de la composition est associée aux genres en prose plus proches des pratiques sociales, et la poésie semble concentrer en elle toutes les potentialités de l'invention, préfigurant la fonction poétique jakobsonienne.

22 L'écriture et la prose cèdent du terrain tandis que l'histoire littéraire l'emporte sur la lecture critique sollicitée uniquement pour l'étude des figures.

23 La régulation de ces déséquilibres constitue la principale préoccupation de G. Lanson dans ses Conseils sur l'art d'écrire (1913). Il replace les figures dans la langue et défend l'idée que leur usage original en discours ne saurait résulter de leur application mécanique mais requiert à la fois l'authenticité du sentiment et l'expérience de l'écriture : «Vous pouvez aimer votre vieux jardinier, sans être capable d'écrire ces simples mots : « Maître Paul vient de mourir ; notre jardin en est tout triste » (16), dit-il en citant une personnification célèbre de $\mathrm{M}^{\mathrm{me}}$ de Sévigné. 

l'élocution. Mais il renonce au catalogue et enchaine les figures selon la logique associative qui leur est propre. Ainsi il commence par la métaphore pour en tirer ensuite la comparaison comme un stade explicité de la métaphore qui « généralement contient en germe une comparaison» (188). Et il montre que l'emploi d'une figure n'est pas indépendant de celui des autres, qu'elles peuvent se contrarier, s'annihiler ou se renforcer. celui de l'expression écrite, ce qui rejaillit sur le type de lecture préconisé. La lecture personnelle chère à $\mathrm{G}$. Lanson doit nourrir l'écriture. Elle y trouve un prisme d'émotions déjà exprimées par des sensibilités différentes qui l'aident à se décentrer, et des ressources expressives, présentées non comme des recettes, mais sous la forme d'un éventail de possibilités. Le manuel a d'abord été édité dans une collection réservée aux jeunes filles et ouvert par la suite à l'usage des élèves des lycées et collèges et de l'enseignement primaire supérieur. «En écrivant pour les jeunes filles, j’ai écrit pour tout le monde, car je me suis adressé au jugement, à la raison, qui sont en elles comme en nous » (VI) revendique l'auteur dans une préface ultérieure. Pourtant, son choix de revenir aux objectifs communicationnels et expressifs de la rhétorique ancienne pour faire accéder un public non lettré à la maitrise des discours sociaux n'a pas suscité autant d'émules qu'on aurait pu s'y attendre quand il s'est agi de démocratiser l'enseignement secondaire.

\section{La didactique des figures au collège}

Dans la première moitié du $\mathrm{xx}^{\mathrm{e}}$ siècle, la résistance des humanités classiques provoque le rejet des humanités modernes (réforme de 1925), et le retour du terme "rhétorique» dans les Instructions de 1935 (Houdart-Mérot, 28). Mais dans les contenus comme les compétences, la littérature triomphe définitivement de la rhétorique. L'histoire littéraire revient dans les programmes en 1935. En 1938, «De la sixième à la classe de Première, pour l'explication française comme pour la lecture suivie, le programme est composé exclusivement de textes littéraires" (op.cit., 29). La rhétorique subit une double réduction. Côté production, on n'apprend plus à écrire des textes originaux, mais des écrits métatextuels, explication, commentaire, dissertation. Côté réception, l'explication de textes se centre sur les seules microstructures qu'elle met en rapport avec les intentions de l'auteur, solution qui évite de faire appel aux registres, genres et types de discours enseignés par les rhéteurs.

La création du collège unique en 1975 entraine la suppression du primaire supérieur et la mise en continuité du primaire inférieur avec le secondaire inférieur, première étape vers la démocratisation du secondaire supérieur, qui perdure dans la situation actuelle, bien que le maintien du collège unique soit régulièrement débattu.

\subsection{Les figures dans les programmes}

L'évolution des figures dans les programmes du collège est étroitement liée à celle des politiques éducatives qui ont oscillé entre un modèle ascendant, visant les apprentissages communicationnels fondamentaux, et un modèle descendant, visant la transmission d'une culture patrimoniale à dominante littéraire. Elle a également été influencée par les répercussions des avancées des sciences humaines sur l'enseignement. La refondation de 
la rhétorique restreinte réhabilite les figures comme marques de littérarité (le Groupe $\mu$ publie sa Rhétorique générale en 1970, sa Rhétorique de la poésie en 1977, son Traité du signe visuel en 1992). La refondation de la rhétorique ancienne redonne vigueur aux apprentissages argumentatifs (C. Plantin publie ses Essais sur l'argumentation en 1990). Simultanément, la linguistique du discours (Kerbrat-Orecchioni, 1980) et la linguistique textuelle (Adam, 1997) inventent des outils de modélisation à priori des textes qui viennent concurrencer la modélisation empirique de la poétique des genres. La didactique du français, apparue comme toutes les didactiques dans le sillage des sciences de l'éducation, va devoir se tracer une route au milieu de ce large choix d'options.

Les programmes et accompagnements de 1999 mettent en place le décloisonnement des sous-disciplines du français autour de la lecture analytique :

«Observer un texte pour un élève de collège signifie s'arrêter sur des éléments présents dans le texte et identifier des relations à distance entre ces éléments : parallélismes; contrastes, oppositions, similarités, etc. Ces observations portent aussi bien sur les formes d'expression (faits de langue lexicaux et grammaticaux, construction de phrase, rythmes et sonorités) que sur celles du contenu (thèmes, figures de style, éléments d'intertextualité, etc.)» (97).

Le passage de la lecture linéaire à la lecture tabulaire améliore la perception du fonctionnement des figures, mais embrouille leur désignation en surévaluant les figures du signifié et en leur subordonnant celles du signifiant, ce qui conduit à évincer ces dernières de l'extension du terme. En outre - et c'est là le point d'achoppement le plus sensible de la transposition didactique de la rhétorique restreinte par la lecture analytique -, le seul effet des figures retenu est le soulignement du sens, alors que les rhétoriciens modernes ont mis en valeur leur effet antireprésentatif.

31 Les figures dites "essentielles»: comparaison, métaphore, métonymie, antithèse, symbole, périphrase (124) sont enseignées au cycle central $\left(5^{\mathrm{e}}-4^{\mathrm{e}}\right)$, et leur rôle de médiateur de la lecture-écriture réaffirmé : «À travers l'exploration des figures, ce sont les capacités de élèves qui sont développées, notamment à l'écrit: jeux de langage, créativité verbale, fonctionnement associatif, recherche des effets sur le lecteur et l'auditoire » (ibid.). Le figement devient un critère contrastif d'évaluation de la créativité littéraire : « au terme du cycle central, les élèves distinguent les figures figées par l'usage et les figures créatrices de significations nouvelles » (ibid.).

L'introduction de la lecture de l'image dans les programmes est à la fois une conséquence du développement de la sémiotique (Barthes) et de la rhétorique (Groupe $\mu$ ) de l'image, et un moyen de compenser les difficultés de lecture de certains élèves, pour lesquels l'image fixe et le film facilitent l'accès aux œuvres étudiées. On se sert donc de l'image pour faciliter le repérage des figures, notamment l'ironie, avec l'étude du dessin d'humour.

En $3^{\mathrm{e}}$ on ajoute aux figures de ressemblance les « figures d'addition, comme les gradations ou les parallélismes, les figures d'atténuation, comme l'euphémisme ou la litote, et les figures d'opposition » (171). L'apprentissage de l'argumentation rétroagit sur celui des figures par le biais de l'axiologie : « Les figures de style [...] peuvent se charger d'une forte valeur axiologique: "Il s'est battu comme un lion" s'oppose à "il s'est enfui comme un rat" " (200). Toutes les disciplines pratiquent l'argumentation. On reconnait donc aux figures un statut interdidactique de jure, même si, dans les faits, l'étude pluridisciplinaire de l'argumentation les prend rarement en compte :

«L'argumentation en classe de français reçoit sa définition de la tradition rhétorique (à la différence de la démonstration scientifique); la notion même 
d'argumentation, sa définition, les modes de son fonctionnement, varie selon les disciplines [...]. La classe de français est le lieu où peut être développée une réflexion pluridisciplinaire sur l'argumentation » (172). des séquences consacrées aux œuvres et groupements de textes de l'année, et un livre de grammaire. précise au moyen de couleurs les parties des leçons qui conviennent à chaque niveau. Cette « somme grammaticale » a influencé les choix grammaticaux et lexicaux de tous les manuels (Magnard et autres).

39 La leçon 31 Les figures de style (141-145) est préparée par trois autres : 38 Le sens des mots (propre/figuré), 29 Le classement des mots d'après leur sens (synonymie/antonymie), 30 Le champ lexical. Les tropes en un mot ne sont pas seulement mis en avant parce qu'ils sont plus frappants et plus simples à analyser que ceux en plusieurs mots, mais aussi parce qu'ils permettent d'articuler apprentissages lexicaux et apprentissages stylistiques, même si ce n'est pas sans risques. En effet, quand les mots appartiennent à un champ lexical par leur sens figuré, la détermination de ce champ occulte la perception des figures. Aussi l'exercice de style Botanique de Queneau : «Après avoir fait le poireau sous un tournesol, merveilleusement épanoui, je me greffai sur une citrouille en route vers le champ Perret, etc. » est-il utilisé pour repérer le champ lexical de la botanique et non les métaphores (137). 

(140). Les effets sont énoncés avec des infinitifs injonctifs qui inscrivent les figures dans une perspective communicationnelle: montrer une ressemblance, exagérer, atténuer, répéter, insister, construire une opposition. Les figures en plusieurs mots sont rattachées sémantiquement et illocutoirement aux figures en un mot: la comparaison à la métaphore, l'euphémisme à la litote. Assonance et allitération sont réintégrées dans les procédés d'insistance, aux côtés de l'anaphore et de la gradation. On a toutefois renoncé pour cette leçon principale à un autre effet fédérateur des figures de style: la modalisation, traitée plus loin (339). Noyées au milieu d'autres structures linguistiques, les figures sont considérées comme des moyens discursifs parmi d'autres. Les exercices assurent une initiation au repérage et à la reproduction mimétique sur des fragments choisis ad hoc.

Si la grammaire a pour fonction de synthétiser les savoirs à retenir, les manuels sont chargés eux de mettre en relation savoirs et compétences (lecture, écriture). Les redondances entre la partie grammaire, le lexique récapitulatif final, les questionnaires de lecture et les exercices de grammaire associés aux séquences, permettent d'associer le fonctionnement des figures comme marqueurs de genre et de registre à leurs définitions et dénominations. Peu importe que ces dernières apparaissent dans une leçon globale (Hatier $5^{\mathrm{e}}$, Magnard $5^{\mathrm{e}}$ Jardin des lettres et Rives Bleues), des leçons spécifiques (comparaison, métaphore, Didier Fil d'Ariane $5^{\mathrm{e}}$ ) ou des encarts définitionnels (Belin $5^{\mathrm{e}}$ L'œil et la Plume).

L'essentiel est ailleurs: dans l'interprétation des programmes. Les manuels leur sont fidèles, mais s'accordent deux latitudes, celle de la répétition: ils reviennent chaque année sur le «noyau dur des figures» (Herschberg Pierrot, 1993 : 189), les tropes en un seul mot, particulièrement les tropes de ressemblance; et celle de l'adjonction pour peu qu'elle aide la lecture. Ainsi le Jardin des lettres Magnard $6^{\mathrm{e}} 2009$ fait analyser l'antimétabole que Lewis Carroll prête à Alice «Je dis ce que je pense [...] du moins je pense ce que je dis ", sans la nommer, mais en suivant pas à pas la dénonciation par le Chapelier et le Loir du paralogisme qu'elle induit (55) ${ }^{2}$. L'édition de 2013 met fin à cet apprentissage logique en tronquant le passage et en le raboutant à un autre portant sur des devinettes (59) qui semble désavouer les censeurs d'Alice. Choix imputable moins à une baisse d'ambition qu'à la réforme de 2008 qui a introduit l'histoire des arts comme objet interdidactique transversal : l'extrait sert de support à l'analyse d'une séquence de la transposition cinématographique de Tim Burton (2010).

Pour expliquer les variations entre manuels et rééditions du même manuel, il donc faut remonter à l'architecture des programmes qui elle-même guide le choix des supports textuels.

On réalise alors combien l'objet didactique figures de style a gagné en complexité au fil du temps. Désormais, il se trouve à l'intersection de cinq apprentissages : ceux de l'histoire littéraire (parallèle au programme d'histoire : Antiquité en $6^{\mathrm{e}}$, Moyen-Âge en $5^{\mathrm{e}}$, etc.), des archigenres littéraires (poésie, théâtre, récit de fiction, autobiographie), des registres qui permettent de spécifier les sous-genres ou d'apprécier leur métissage, des types de discours qui forment l'épine dorsale de la progression curriculaire (narratif en $6^{\mathrm{e}}$, descriptif et explicatif au cycle central, argumentatif en $3^{\mathrm{e}}$ ), et de l'histoire des arts.

On comprend mieux pourquoi l'hyperbole apparait tantôt en $6^{\mathrm{e}}$ avec l'épisode de Polyphème dans l'Odyssée, tantôt en $5^{\mathrm{e}}$ avec les romans de chevalerie, pourquoi l'étude 
des fables de La Fontaine favorise l'étude de la personnification, celle des métamorphoses d'Ovide la métaphore et la comparaison, et celle de la poésie lyrique en $4^{\mathrm{e}}$ l'antithèse. On ne s'étonne pas que les calembours soient traités à la fois dans les jeux poétiques en $6^{\mathrm{e}}$ et la farce courtelinesque en $5^{\mathrm{e}}$, ce qui laisse apparaitre en filigrane une sixième ligne d'apprentissage : l'orthographe, dont la maitrise est un objectif majeur du collège. préparation à la vie professionnelle des élèv qui atteignentlalinite d’age de obligatoire et la préparation au lycée de tous les autres. L'écart est remarquable entre le minimum affiché par les programmes, limité à l'identification et la nomination d'une douzaine de figures, et l'objectif poursuivi par les manuels et les enseignants: aider l'élève à construire un projet de lecture personnel et adapté pour chaque texte. Cette seconde perspective sort du cadre de la rhétorique traditionnelle, qui connecte des listes de situations d'emploi et de figures, pour aborder celui de la rhétorique générale, qui couvre l'ensemble des mécanismes figuraux, au moyen des quatre opérations cognitives fondamentales : adjonction suppression, substitution, permutation. On trouve l'ellipse traitée aussi bien au niveau phrastique qu'au niveau narratif, associée à la prolepse et l'analepse, toutes les trois étant qualifiées de procédés (Magnard $3^{\text {ème }}, 324$ ). Une telle approche fait pendant au projet scientifique de Dupriez qui a sous-titré son dictionnaire Gradus, les procédés littéraires. En réunissant toutes les figures, traditionnelles et récentes, et toutes leurs dénominations, avec des exemples modernes et classiques, celui-ci a voulu faciliter l'approche personnelle des figures, par quelque bout qu'on les prenne.

51 Comme le lexicologue, les auteurs de manuels cherchent à faire apprendre les figures non pour elles-mêmes mais pour les effets qu'elles permettent, et dans une perspective non 
seulement de réception mais de production ${ }^{4}$. Toutefois, ils doivent se plier aux contraintes de la transposition didactique. Ils simplifient la réalité. L'énoncé «Expliquez dans l'incipit de $W$ de Perec, l'expression "l'Histoire avec sa grande Hache" ? De quelle figure de style s'agit-il» (Magnard $3^{\mathrm{e}}$, 94) montre que la réponse : personnification, est programmée à la fois par l'apprentissage précoce des figures d'analogie, et par la volonté délibérée de ne pas traiter les superpositions de figures, ici calembour (entre le h et la hache) sur personnification. Parfois le besoin de simplifier amène à reconstruire la réalité : à expliquer la métaphore comme une comparaison privée de terme comparatif.

Le second trait distinctif de l'action didactique est la surdétermination des objets enseignés. Il s'agit là d'une économie aux deux sens du terme: gain de temps et organisation, mais qui n'est pas sans danger. Les figures dans la poésie engagée (au programme de la $3^{\mathrm{e}}$ ) ne sont pas toutes liées à l'argumentation, de même que les jeux de mots apparaissent ailleurs que dans la poésie. Dans Magnard Jardin des Lettres $6^{e} 2013$, cinq pages séparent la demande d'explication d'un à peu près poétique : «à brise abattue » dans un poème de Claude Roy (129), du calembour contenu dans le titre du chapitre : Enée et Ulysse, deux héros à Troie ( = trois). Si les auteurs n'ont pas attiré l'attention des lecteurs sur leur calembour, c'est peut-être qu'il était involontaire, ou qu'ils ne voulaient pas troubler le délicat équilibre entre registre sérieux et registre ludique. Cela aurait été pourtant une occasion de réintégrer dans la pratique figurale le discours du manuel, un moyen aussi de rappeler que les figures existent en dehors de la littérature.

\section{Conclusion}

53 Les figures de style sont un point d'ancrage fort de l'identité de la discipline français. Elles ont cristallisé autour d'elles l'héritage de la rhétorique, dont le retour dans les programmes à partir des années 1980 s'explique non seulement par l'évolution des sciences humaines, et notamment l'apparition de la linguistique du discours, mais aussi par la coïncidence entre les valeurs de l'école républicaine (égalité de traitement pour tous dans l'acquisition du lire-écrire-parler) et les valeurs antiques (donner au citoyen la possibilité de se défendre et de s'exprimer).

Il n'y a pas eu de solution de continuité dans l'enseignement des figures depuis l'époque classique, époque à laquelle la didactique du français au sens d'apprentissage raisonné et institutionnalisé de la langue et du discours en langue française a vu le jour. Ceci implique des traditions vivaces, qu'il faudrait plus souvent interroger. Ainsi A. Herschberg Pierrot (1993 : 191) a montré que l'exemple canonique de la litote emprunté à la réplique de Chimène dans Le Cid «Va, je ne te hais point » était faux, puisque celle-ci, resituée dans son cotexte se révèle être une simple reprise de la réplique précédente. Les manuels puisent désormais leurs exemples dans les scénarios de la vie quotidienne : «Tu n'es pas vraiment un cordon-bleu » (Magnard 3 ${ }^{\mathrm{e}}, 336$ ).

C'est aussi à l'époque classique qu'il nous a fallu remonter pour expliquer l'omniprésence de la littérature dans l'enseignement du français langue première, et le rôle particulier des figures dans l'accrochage des extraits littéraires aux apprentissages discursifs. L'exemplification des figures est un des premiers sinon le premier exemple de surdétermination didactique dans l'histoire de la discipline. Elle a introduit la littérature dans les programmes tout en convertissant les textes littéraires en supports d'apprentissage polyvalents. Dans le système scolaire actuel, on peut donc tout apprendre 
des figures, ou plus généralement de la langue et du discours, sans (presque) sortir du discours littéraire.

Nous conclurons donc notre propos, comme il est d'usage pour une étude didactique, sur deux préconisations. Il nous semble indispensable de renouer avec l'orientation qui était celle des premiers manuels du collège unique : associer par la comparaison la littérature et les autres discours sociaux, à l'instar de Mots et merveilles Magnard $5^{\mathrm{e}}$ (1982) qui mettait en parallèle l'emploi des figures de style dans le journalisme sportif et l'épopée ${ }^{5}$. Il serait également souhaitable de développer les occasions de comparer les figures dans diverses langues, en étudiant les différences entre original et traduction, alors que l'on fait souvent étudier les figures sur des textes traduits, comme s'ils étaient écrits en français, et en sollicitant les compétences plurilingues des élèves, ce qui est une pratique courante en didactique des langues étrangères. Présentes dans de nombreux proverbes et expressions idiolectales, les figures sont un embrayeur d'apprentissages interculturels qui ne demande qu'à être exploité.

\section{BIBLIOGRAPHIE}

ADAM, J.-M. (1997) : Les textes : types et prototypes/Récit, description, argumentation, explication et dialogue, Paris, A. Colin.

BoIssinot, A. (2001) : «Introduction », in : A. Boissinot, A. Arnaud \& J. Jordy (coords), Profession enseignant. Le français en collège et en lycée, Paris, Hachette, p.10-17.

DUPRIEZ, B. (1984) : Gradus. Les procédés littéraires (dictionnaire), Paris, Union générale d'éditions. GENETTE, G. (1977) : «Introduction », in : P. Fontanier, Les Figures du discours, Paris, Flammarion, p. 6-17.

GAILLARD, G.-H. (1822) [1745] : Rhétorique française, à l'usage des jeunes demoiselles avec des exemples tirés, pour la plupart, de nos meilleurs orateurs et poètes modernes, Paris, Depélafol libraire.

GROUPE $\mu$ (1970a) : Rhétorique générale, Paris, Larousse.

- (1970b) : Rhétorique de la poésie, lecture linéaire, lecture tabulaire, Bruxelles, Complexe.

- (1992) : Traité du signe visuel. Pour une rhétorique de l'image, Paris, Éd. Le Seuil.

HERSCHBERG Pierrot, A. (1993) : Stylistique de la prose, Paris, Belin.

HOUDART-MÉROT, V. (2001) : «Des humanités à la fracture des années soixante », in : A. Boissinot, A. Arnaud \& J. Jordy (coords), Profession enseignant. Le français en collège et en lycée, Paris, Hachette, p. 28-32.

KERBRAT-ORECCHIONI, C. (1980) : L'Énonciation de la subjectivité dans le langage, Paris, A. Colin.

LAMY, B. (1747) [1675] : La Rhétorique ou l'art de parler, Paris, Didot.

LANSON, G. (1913) : Conseils sur l'art d'écrire. Principes de composition et de style, Paris, Hachette.

MESTRE, le P., S. J. (1902) [1882] : Principes de littérature, style, composition, poétique, histoire littéraire des genres, Paris, Delhomme et Briguet. 
MAURON, C. (1963) : Des Métaphores obsédantes au mythe personnel. Introduction à la psycho-critique,

Paris, J. Corti.

PLANTIN, C. (1990) : Essais sur l'argumentation, Paris, Kimé.

ROUSSEAU, J.-J. (1969) [1762] : CEuvres complètes IV, Paris, Gallimard.

VIALA, A. (1985) : Naissance de l'écrivain, Paris, Éd. de Minuit.

\section{ANNEXES}

\section{Textes officiels}

Enseigner au collège, FRANÇAIS, français, Programmes et Accompagnement, CNDP, MENRT, juillet 1999 (édition précédente mars1999).

B.O. $\mathrm{n}^{\circ} 28 \mathrm{du} 15$ juillet 2004, Programmes d'enseignement du français $6^{\mathrm{e}}, 5^{\mathrm{e}}, 4^{\mathrm{e}}, 3^{\mathrm{e}}$, MEN.

Livret personnel de compétences de l'élève, Attestation de maîtrise des connaissances et compétences du socle commun, MEN, 2007.

B.O. spécial $\mathrm{n}^{\circ} 6$ du 20 août 2008, Programmes du collège, Programmes de l'enseignement du français $\left(2009-10: 6^{\mathrm{e}} ; 2010-11: 5^{\mathrm{e}}, 2011-12: 4^{\mathrm{e}} ; 2012-13: 3^{\mathrm{e}}\right)$. MEN.

Manuels

Nouvelle GRAMMAIRE du collège, $6^{e}, 5^{e}, 4^{e}, 3^{e}$, Magnard, collection dirigée par Georges

Molinié, 2007.

Français $6^{e}$, Magnard, Jardin des lettres, 2009.

Français $6^{e}$, Magnard, Jardin des lettres, 2013.

Français $6^{e}$, Hatier, Colibris, 2014.

Mots et Merveilles, Magnard 5e , Louis Arnaud, Anne Garrigue, 1982.

Français $5^{e}$, Magnard, Jardin des lettres, 2010.

Français 5 $5^{\mathrm{e}}$, Magnard, Mots et émotions, 2010.

Français 5 $5^{e}$, Belin, L'œil et la plume, 2010.

Français $5^{e}$, Hatier, Rives bleues, 2010.

Fil d'Ariane 5 ${ }^{e}$, Didier, 2010.

Français $4^{e}$, Magnard, Jardin des lettres, 2011.

Français $3^{e}$, Magnard, Jardin des lettres, 2012.

\section{NOTES}

1. Le nombre des principales figures de style rassemblées dans le tableau récapitulatif du Magnard $3^{\mathrm{e}}(2012: 431)$ sont les suivantes : anaphore, antithèse, comparaison, euphémisme, gradation, hyperbole, litote, métaphore, métonymie, oxymore, périphrase, personnification. 
2. «Récris dans un tableau à deux colonnes les doubles affirmations d'Alice, du Chapelier et du Loir. Par quel procédé passe-t-on chaque fois de la première affirmation à la seconde? ». Ce procédé est la réduplication de la figure par les contradicteurs : «je vois ce que je mange, je mange ce que je vois» (Chapelier), «je respire quand je dors, je dors quand je respire» (Loir). Reprenant la définition de l'antimétabole de Littré : «Deux phrases font l'échange des mots qui les composent de manière que chacun se trouve à son tour à la même place et dans le même rapport où était l'autre ». B. Dupriez $(1984,54)$ signale qu'elle est «propre à remettre en cause les liens de causalité ».

3. « Avis », extrait de Au rendez-vous allemand, 1945.

4. B. Dupriez rappelle sur la page de titre que Gradus est l'abrégement de Gradus ad Parnassum, nom générique « aux siècles classiques des manuels qui facilitaient la composition littéraire ».

5. «Relevez les comparaisons, les images et les qualificatifs utilisés [...] pour nommer et caractériser les joueurs. Montrez que, comme dans l'épopée [...] ou la chanson de geste ( $\mathrm{La}$ Chanson de Roland, p. 55), le compte rendu ou l'essai sportif chante la gloire des "héros" modernes » (p. 131).

\section{RÉSUMÉS}

Léguées par la tradition rhétorique, les figures de style sont un des objets didactiques les plus anciens de la discipline français. Leur étude au collège est marquée par le clivage entre leur connotation patrimoniale littéraire et leur fonction dans l'outillage de la lecture et de la production écrite. L'étude est organisée en deux parties: la première, diachronique, suit l'évolution de la didactique des figures $\mathrm{du} \mathrm{XVII}^{\mathrm{e}}$ au XX $\mathrm{XX}^{\mathrm{e}}$ siècle en parallèle avec la mise en place de l'institution scolaire; la seconde, synchronique, montre la place actuellement occupée par les figures dans l'enseignement du français au collège en France.

Abstract: Bequeathed by the rhetorical tradition, the figures of speech are one of the oldest learning objects of the French first language. Their study in high school is marked by the split between their literary heritage connotation and their function as tools of reading and written production. Our study is organized in two parts: the first, diachronic, follows the evolution of the teaching of rhetorical figures from the seventeenth to the twentieth century in parallel with the establishment of educational institution; the second, synchronic, shows the place currently occupied by the rhetorical figures in the teaching of French in high school in France.

\section{INDEX}

Mots-clés : figure, collège, langue, discours, littérature

Keywords : rhetorical figure, secondary school, language, discourse, literature

\section{AUTEUR}

\section{NICOLE BIAGIOLI}

I3DL (EA 6308), Université Nice Sophia Antipolis 